\title{
Il était une fois... la formation à distance
}

\section{Once upon a time... distance learning}

\section{Érase una vez... la formación a distancia}

\author{
RESPONSABLE DU NUMÉRO \\ Viviane Glikman, enseignante-chercheuse \\ Membre du Gehfa (Groupe d'études - histoire de la formation des adultes), France \\ viviane.glikman@free.fr
}

\section{RÉSUMÉ}

Cet éditorial propose plusieurs angles sous lesquels il est possible d'aborder l'histoire de la formation à distance. Si l'histoire des technologies est sans doute la plus souvent retracée, d'autres histoires sont ici identifiées : celle des pédagogies, celle des contextes de toute nature qui environnent les dispositifs et leur évolution, celle des institutions spécialisées dans ce domaine et, enfin, celle de la terminologie utilisée pour nommer les actions réalisées. Dans un second temps sont présentés les différents articles qui composent ce numéro, adoptant un ou plusieurs des angles considérés.

Mots-clés : formation à distance, formation en ligne, histoire, technologies, pédagogie, contexte sociétal, institutions

\section{ABSTRACT}

This editorial presents different views to approach the history of distance learning. If the history of technologies is the most frequently explored, this paper also presents the history of pedagogies and the various contexts surrounding devices and their evolution, specialized institutions in the field and the terminologies used to name different actions. Subsequently, it presents the papers of the current issue that adopted some of these views on the topic.

Keywords: distance learning, online learning, history, technologies, pedagogy, societal context, institutions 
Este editorial sugiere muchos ángulos de visión a través de los cuales es posible abordar la historia de la educación a distancia. Aunque la historia de las tecnologías es la que se trata más a menudo, en este trabajo se identifican otras historias, como la de las pedagogías, la de los contextos alrededor de los entornos y de su evolución, la de las instituciones especializadas en el ámbito $\mathrm{y}$, por último, la de la terminología utilizada para nombrar los proyectos que se llevan a cabo. A continuación, se presentan los distintos artículos que constituyen este número a partir de una o de varias de las perspectivas consideradas.

Palabras clave: formación a distancia, aprendizaje en línea, historia, tecnologías, pedagogía, contexto social, instituciones

Pourquoi un numéro thématique de la revue Médiations et médiatisations sur l'histoire de la formation à distance?

Parce que de nouvelles expérimentations en matière de formation à distance apparaissent en permanence, sans toujours tenir compte des tentatives précédentes et, parfois même, sans que leurs auteurs en aient connaissance. Parce ces expérimentations, souvent conçues dans l'enthousiasme, se fondent sur les potentialités sans cesse accrues des technologies de l'information et de la communication, dites " nouvelles » au fur et à mesure de leur apparition, depuis la fin du XXe siècle, jusqu'au numérique et aux réseaux. Elles tentent certes de renouveler les modes d'enseignement et d'apprentissage, mais prennent aussi le risque de confondre innovation technologique et innovation pédagogique. Certains acteurs institutionnels multiplient les ressources disponibles et tentent d'optimiser les interactions entre les apprenants et entre les apprenants et leurs tuteurs, dans l'espoir d'atteindre in fine des taux de persévérance et de réussite plus élevés; ils élaborent ainsi des dispositifs complexes ou sophistiqués à la « transparence » illusoire qui, à terme, n'atteignent qu'imparfaitement leurs objectifs.

II semble être temps, aujourd'hui, de s'interroger sur l'histoire fertile d'expériences qui perdurent, sur leurs places dans des dispositifs de formation dominés par le présentiel et sur leurs effets sur les apprentissages, dans l'espoir d'alimenter la réflexion de ceux qui s'investissent ou désirent s'investir dans ce champ en connaissance de cause. II ne s'agit donc nullement de décourager de nouvelles tentatives, bien au contraire, mais de proposer, plus modestement, de tenir compte des acquis dans leur élaboration.

\section{Une histoire ou des histoires de la formation à distance?}

Plusieurs facteurs se sont imbriqués au fil des décennies pour influencer les évolutions de la formation à distance. De leur interpénétration sont issues des modalités nouvelles, parfois innovantes, d'apprentissage. Deux de ces facteurs dominent les approches; ils concernent l'histoire des technologies et celle des pédagogies. Ces histoires s'inscrivent néanmoins dans celle des contextes socioéconomiques et éducatifs ainsi que dans celle des institutions. De plus, la terminologie a évolué au fil du temps, s'adaptant aux circonstances. Chaque nouveau dispositif de formation à distance a émergé à un moment donné de ces histoires du temps présent, se situant au confluent de ces divers facteurs, et, lorsqu'il existe de longue date, s'est déployé, parfois modifié, au fur et à mesure du temps. Ce sont ces processus, leurs causes et leurs effets, que nous souhaitions voir traiter dans ce numéro. 
Les études et recherches dans le domaine de la formation à distance sont riches et variées ${ }^{1}$. Beaucoup interrogent les technologies et rapportent des expérimentations de multiples dispositifs, dont elles montrent tantôt la pertinence, tantôt l'incertaine efficacité. D'autres travaux s'intéressent aux pédagogies de la distance (Dessus et Marquet, 2009; Lameul et Loisy, 2014; Poteaux, 2013). D'autres encore concernent les acteurs, qu'ils soient enseignants (Baron, 2010; Boissoneault, 2009; Papi, 2016), tuteurs (Depover et al., 2011) ou apprenants (Deschênes, 1999; Glikman, 1999), les politiques institutionnelles (JacquinotDelaunay et Fichez, 2008; Campus France, 2020), etc. L'histoire n'y est souvent présente que circonstanciellement ou comme toile de fond, mais il arrive qu'elle constitue l'objet même de publications (Albero, 2004; Audet, 2012; Baron et Depover, 2019; Chaptal, 1995; CLIFAD, 2007), qui apportent à leur tour de très utiles éléments de réflexion. Ce n'est donc pas la première fois qu'une telle approche est entreprise, mais ce moment particulier du recours à la distance pour des raisons sanitaires nous est apparu propice à un retour sur des usages plus pérennes.

$\mathrm{Si}$ « le passé est la lanterne du futur » (Mazouz, 2015), on peut espérer que ce numéro nous aidera à répondre à des questions fondamentales : dans quelle mesure et à quelles conditions les formations à distance ont-elles réellement offert et peuvent-elles continuer d'offrir la « liberté d'apprendre » à son choix et à son rythme, liberté que des organismes spécialisés affirment souvent permettre, aussi bien en France (Centre national d'enseignement à distance, Conservatoire national des arts et métiers) qu'au Québec (Télé-université du Québec, devenue Université TÉLUQ)? Dans quelle mesure et à quelles conditions ontelles véritablement ouvert et peuvent-elles continuer d'ouvrir l'accès aux connaissances et aux certifications à des publics qui en étaient jusque-là exclus, c'est-à-dire contribuer à l'idéologie démocratique que revendiquent les institutions qui les portent sans se contenter de tendre "à favoriser avant tout ceux qui le sont déjà et à "faire pleuvoir là où c'est mouillé" »(Glikman, 2002, p. 266)?

La première histoire, celle des technologies dont l'évolution chronologique, commune à tous les pays occidentaux et au-delà, est celle qui peut le plus aisément donner lieu à une périodisation temporelle, base de toute interprétation historique et fondement pour l'analyse et la compréhension des processus à l'œuvre (Le Goff, 2014). II n'en reste pas moins que ce découpage technologique du temps n'exclut ni une continuité relative, et souvent provisoire, dans l'usage des supports, ni une influence significative des autres facteurs.

\section{L'histoire des technologies}

Qu'on la fasse remonter au milieu du XIX ${ }^{e}$ siècle, comme la plupart des auteurs, à commencer par Henri Dieuzeide (1985), ou, plus étonnement, à l'aube du christianisme, comme l'affirme John Daniel dans ce numéro, la formation à distance a connu nombre d'avatars subordonnés aux supports disponibles, participant désormais aux phénomènes d'industrialisation à l'œuvre dans la formation (Guillemet, 2004; Mœglin, 2016). À l'imprimé des cours par correspondance est venu s'adjoindre l'audiovisuel avec des émissions de radio et de télévision éducatives, plus tard supplantées par les cassettes audio et vidéo, les «multimédias » combinant divers moyens (Glikman et Baron, 1991), puis l'informatique avec l'Enseignement assisté par ordinateur (EAO) et, maintenant, le numérique et les réseaux avec les formations en ligne qui combinent toutes ces approches sur un même écran unique via des plateformes dédiées. À partir de la succession de ces technologies, on peut distinguer quatre grandes périodes dans l'histoire de la formation à distance : le temps des cours par correspondance (1850-1960), la prédominance de l'audiovisuel (1961-1980), l'hégémonie de l'informatique (1981-2000) et la suprématie des formations en ligne (2001 à nos jours) (Glikman, 2016).

\footnotetext{
${ }^{1}$ Tant de textes sur la formation à distance ont été publiés au cours des précédentes décennies que les quelques publications citées en référence ne sont ici que pour illustrer notre propos, sans prétendre à une quelconque exhaustivité, ni même à une sélection fondée sur leur importance.
} 
Même si le cycle enthousiasme/désillusion, analysé depuis longtemps par Larry Cuban (1986), ne cesse de s'appliquer au fur et à mesure des innovations technologiques et si chaque nouvelle technologie a été ambitieusement annoncée comme porteuse d'une " révolution » pédagogique, il semble être aujourd'hui admis que seuls comptent le désir et la volonté de ceux qui souhaitent mettre efficacement les moyens techniques au service de l'accès au savoir du plus grand nombre et de la qualité des apprentissages.

Certes, l'approche par les technologies, à elle seule, est insuffisante pour répondre à la question de savoir dans quelle mesure ces changements ont ou non modifié les actes d'apprendre et d'enseigner à distance et c'est l'histoire des pédagogies qu'il convient alors de convoquer.

\section{L'histoire des pédagogies}

Les nombreuses recherches et expérimentations sur les méthodes de la pédagogie en général et de la pédagogie universitaire à distance en particulier ont alimenté les formations à distance et leurs approches. Le modèle transmissif (ou béhavioriste) véhiculé par l'imprimé et le courrier postal a dominé pendant de nombreuses décennies; le modèle cognitiviste, fondé sur le "conflit cognitif», déséquilibre puis accommodation entre les représentations des apprenants et les données nouvelles, a ensuite pénétré les approches pédagogiques proposées. Nombre de formations à distance privilégient désormais le constructivisme, qui met l'accent sur l'activité des apprenants dans la construction des connaissances au sein d'un ensemble de ressources, et le socioconstructivisme, qui rend possible la collaboration entre apprenants et contribue de la sorte à rompre le sentiment d'isolement, en reconstruisant virtuellement des communautés d'apprentissage à l'aide des communications via Internet et favorisant ainsi l'instauration d'un lien social. Simultanément, des dispositifs hybrides articulant de manière cohérente des séquences en présence sur site et des périodes de distance recourant à des environnements technologiques, largement évoqués ici par Daniel Peraya dans l'entretien sur l'histoire de l'unité TECFA, correspondent à des approches pédagogiques innovantes (Charlier et al., 2006). Par ailleurs, le connectivisme, théorie de l'apprentissage développée par Georges Siemens (2005) et Stephen Downes et basée sur les apports des nouvelles technologies dans la construction des connaissances, est particulièrement mis en avant dans les MOOC.

Un aspect de la pédagogie, peu abordé ici et dont l'histoire reste à écrire, est celui de l'accompagnement - ou tutorat - dont les modalités se sont également adaptées aux avancées technologiques, recourant d'abord au courrier postal et au téléphone, les possibilités réduites de ces supports limitant les échanges. C'est avec les « médias interactifs " que l'on assiste à un accroissement significatif, car leurs potentialités " vont ouvrir la porte à une prise en charge beaucoup plus précise et permanente du tutorat " (Depover et Quintin, 2011, p. 40), grâce aux interactions tant synchrones qu'asynchrones qu'ils permettent non seulement entre tuteurs et apprenants, mais aussi entre pairs (Papi, 2013).

\section{L'histoire des contextes socioéconomiques et éducatifs}

Les contextes socioéconomiques et éducatifs ont également joué un rôle significatif dans l'évolution de la formation à distance. Nous n'en évoquerons ici que deux exemples. En France, c'est d'abord une éducation permanente hors temps de travail régie par les lois sur la promotion sociale et la formation professionnelle $\left(1959,1966^{2}\right)$ et laissée à l'initiative personnelle des individus et à la bonne volonté des employeurs, dans laquelle les émissions télévisées rejoignaient les téléspectateurs à domicile dans leur

${ }^{2}$ Sur ces lois, voir Benoist (2004). 
temps libre. En 1971, une loi sur une « formation professionnelle continue $»^{3}$ sur le temps de travail soumet une large part de la formation des adultes à un financement par les entreprises, mais la lutte contre le chômage vient l'assujettir dès que la crise économique, engendrée par les chocs pétroliers de 1973 et 1979, menace de plus en plus la situation de l'emploi. La formation à distance apparaît alors comme un moyen de réduire les coûts de personnels et de locaux. Permettant de répartir les activités d'apprentissage sur des lieux différents et à des moments différents (temps de travail et temps de loisirs), elle s'inscrit dans l'infléchissement des pratiques de la formation professionnelle continue vers le "co-investissement » du salarié et de l'entreprise. II faudra néanmoins attendre de longues années avant que les formations à distance soient reconnues par les instances ministérielles comme relevant du financement prévu par la loi, des présences dûment constatées sur site continuant d'être exigées pour justifier la rémunération des périodes de formation - ce qui a favorisé le développement des formations hybrides.

En Grande-Bretagne, c'est l'avènement d'un gouvernement travailliste qui, face au coût très élevé des études universitaires réservées presque uniquement à une élite, a décidé la création d'une université à distance au départ financée par l'État, financement remis plus tard en cause par le gouvernement conservateur. En Amérique du Nord, l'importance des distances géographiques, qui excluait de l'enseignement supérieur les populations éloignées des campus, a présidé à la naissance d'universités à distance destinées à élargir l'accès aux études supérieures à la fois dans une perspective de démocratisation et de recrutement d'une nouvelle clientèle.

Enfin, d'une formation à distance dont la place demeure marginale par rapport au face-à-face inhérent à l'organisation traditionnelle des systèmes de formation, on est passé, tout récemment, en 2020 , à une distance largement répandue et contrainte par la pandémie de COVID-19 au niveau des enseignements tant scolaires que supérieurs. Cette expérience impromptue a pris de court les enseignants qui, pour la plupart, étaient peu familiers avec cette approche et se sont contentés de transférer à la distance les méthodes du présentiel, recourant surtout à des visioconférences magistrales, au grand désarroi de beaucoup d'élèves et d'étudiants, sans compter les difficultés d'accès au numérique pour certains et le manque de convivialité durement ressenti par beaucoup d'entre eux ${ }^{4}$.

\section{L'histoire des institutions}

La formation à distance a peu à peu été adoptée dans de nombreux organismes traditionnels de formation, notamment au niveau universitaire, comme en témoignent, en France, les Centres de télé-enseignement universitaires (CTU) regroupés dans la Fédération interuniversitaire de l'enseignement à distance (FIED) créée en 1987 (Acheré, 2014). Plusieurs départements universitaires eux-mêmes développent des offres de formation à distance, dont l'article sur FORSE (cf. infra) donne un exemple marquant. La situation française est toutefois particulière : bien que cette possibilité ait souvent été envisagée, il n'y existe pas d'université à distance, mais un organisme spécialisé dans ce domaine créé au milieu du $X X^{e}$ siècle qui, après avoir changé de nom à plusieurs reprises, s'appelle aujourd'hui le CNED, Centre national d'enseignement à distance (Bourrel et al., 2008) et intervient à tous les niveaux, du primaire au supérieur.

Cependant, de nombreuses institutions, notamment (mais pas uniquement) universitaires, tant publiques que privées, sont spécialisées dans la formation à distance à travers le monde; les " méga-universités ", analysées il y a plus de vingt ans par Sir John Daniel (1996), en sont des figures emblématiques, mais la

\footnotetext{
${ }^{3}$ Loi $\mathrm{n}^{\circ} 71-575$ du 16 juillet 1971 portant organisation de la formation professionnelle continue dans le cadre de l'éducation permanente. Journal offciel de la République française du 17 juillet 1971. https://www.legifrance.gouv.fr/loda/id/JORFTEXT000000687666/

${ }^{4}$ Les étudiants régulièrement inscrits dans de très coûteuses universités américaines se plaignent d'ailleurs des formations entièrement en ligne imposées par la situation sanitaire, qu'ils qualifient d'expérience universitaire " dégradée », et réclament en justice un remboursement partiel de leurs frais d'inscription (Le Bars, 2020).
} 
réalité est plus multiforme. En ce qui concerne les universités européennes, l'Association européenne des universités d'enseignement à distance (EADTU), réseau institutionnel également créé en 1987, rassemble à la fois une dizaine d'universités d'enseignement à distance et des associations nationales d'universités traditionnelles organisant un enseignement à distance. Beaucoup d'universités nord-américaines offrent également à la fois des cours sur campus et des cours à distance, comme, parmi les plus réputées, l'Université de Boston et celle de Berkeley en Californie.

L'histoire de ces institutions est riche d'enseignements quant à l'évolution et à l'image de la formation à distance dans l'univers de l'enseignement supérieur. On verra dans les entretiens qui s'y rapportent que si certaines universités, comme l'Open University du Royaume-Uni, sont parvenues au fil du temps à se créer une excellente réputation, d'autres, telles que l'Université TÉLUQ au Québec, se sont, récemment encore, heurtées à quantité de préjugés de la part des communautés universitaires traditionnelles. En ce sens, une caractéristique importante du CNED français est qu'il ne délivre pas de diplôme et ne fait que préparer aux diplômes nationaux, ce qui évite que soit immédiatement identifiée l'institution de formation fréquentée par un diplômé. Cela peut être considéré comme un avantage dans la mesure où les employeurs, tout comme des enseignants et dirigeants d'universités traditionnelles, ont parfois des difficultés à admettre que les institutions de formation à distance sont aussi performantes que celles qui donnent la priorité à la présence sur site.

\section{L'histoire de la terminologie}

On parle aujourd'hui volontiers de la « formation à distance » (FAD), vocable assez général que nous avons retenu ici, mais plusieurs termes se sont succédé pour la désigner, recouvrant une réalité évolutive. Les « cours par correspondance » font bien entendu référence au temps où le courrier postal était le seul véhicule de diffusion des cours et des devoirs des apprenants. Cette expression a fait place au milieu du $X X^{e}$ siècle à l'« enseignement à distance " (EAD) qui marque l'emploi d'autres supports de diffusion, surtout audiovisuels, mais ce terme qui privilégie l'émission des connaissances a bientôt été, dans les organismes soucieux de donner une image moins directive de leur action, supplanté par celui de " formation à distance » qui, selon F. Henri, « recouvre sémantiquement les concepts de l'enseignement à distance et de l'apprentissage à distance " (Henri et Kaye, 1985, p. 8).

D'autres termes sont apparus, tels que, à partir des années 1990, "formation ouverte et à distance " (FOAD), qui insiste tantôt sur la souplesse des dispositifs dans leur organisation spatiale et temporelle, tantôt, essentiellement dans le contexte anglo-saxon, sur leur ouverture au plus grand nombre sans condition préalable lors de l'inscription. Le "e-learning " a connu, au début du XXI siècle, un succès grandissant, lié à la mode des anglicismes et au recours à l'informatique. Le terme " formation en ligne » est désormais beaucoup plus souvent utilisé. Enfin, désignant des dispositifs particuliers, l'acronyme MOOC (Massive Online Open Course, traduit le plus souvent en français par « Cours en ligne ouvert et massif », CLOM ${ }^{5}$ ) est maintenant largement diffusé depuis l'apparition de ces cours dans de prestigieuses universités nord-américaines au commencement des années 2000 et le début de leur développement en France et, dans une moindre mesure, au Québec en 2012.

${ }^{5}$ Les recommandations officielles, en France et au Québec, suggèrent plutôt « Cours en ligne ouvert à tous » (CLOT). 


\section{Les textes proposés dans ce numéro}

Malgré leurs nombreuses contraintes liées à la crise sanitaire, les auteurs, dont on trouvera les textes dans ce numéro, ont accepté de répondre à notre appel et de s'abstraire d'un quotidien accaparant pour se projeter dans le passé et contribuer à reconstruire l'histoire de la formation dans son ensemble ou en lien avec des dispositifs dont ils ont été acteurs.

C'est dans l'histoire des technologies que Tony Bates s'inscrit en choisissant de présenter « son » histoire des technologies et des médias dans la formation à distance, prévenant le lecteur que " toute histoire est dans un certain sens personnelle, régie par les centres d'intérêt et l'expérience de ceux qui l'écrivent ". Expert internationalement reconnu, il s'appuie donc sur sa longue expérience anglaise et nord-américaine pour brosser un large panorama de l'histoire de la formation à distance et ses modes de communication, de la communication orale puis écrite jusqu'aux " technologies émergentes " (" réalité augmentée », nouvelles applications de l'intelligence artificielle...), en passant par la vidéo, l'informatique et Internet. Insistant sur le fait que le progrès technologique, même s'il transforme radicalement les processus éducatifs, n'est pas en soi un défi pour l'avenir, il conclut que beaucoup reste à faire en matière d'évaluation et de recherche pour en mesurer les implications.

À la croisée de l'histoire des technologies et de celle des pédagogies, deux articles de recherche s'attachent ensuite à l'histoire de ce qu'il est coutume de nommer des « dispositifs technopédagogiques ".

L'un d'eux, celui de Claire Peltier, sur "La diffusion des cours universitaires en direct », montre que si la transmission en vidéo des enseignements universitaires a servi de substitut au présentiel dans le contexte sanitaire de la pandémie de COVID-19, cette pratique, utilisée depuis longtemps en formation à distance, n'est pas en elle-même une nouveauté. Interrogeant la notion d'innovation, cet article rapporte la mise en place et l'évaluation des effets d'un dispositif de diffusion en direct de trois cours pilotes à l'Université de Genève dans le but de permettre à une partie des étudiants de suivre leur formation sans se rendre sur le campus. II révèle l'intérêt et les limites de cette expérience et signale l'importance d'intégrer à la diffusion des interactions et des activités de la part des étudiants, ce qui pourrait remettre en question les pratiques habituelles des enseignants.

L'autre article, celui de Béatrice Verquin Savarieau, Stéphane Simonian et Jacques Béziat, illustre la situation de la formation à distance dans les universités françaises où l'impulsion des instances ministérielles et des appels à projets assortis de budgets ont permis la création de " campus numériques ", dont, en 2001, FORSE (Formations et ressources en Sciences de l'éducation), campus numérique francophone en sciences de l'éducation. L'analyse de cette expérience et de son histoire souligne en particulier l'importance, pour la pérennité de l'action, non seulement des rapports entre les humains et les objets techniques, mais aussi des relations entre les acteurs de l'action et l'environnement, universitaire en l'occurrence.

Insistant sur le rôle du contexte dans l'article qu'il propose dans la rubrique " Débat », Sir John Daniel, éminent spécialiste du domaine, replace les grandes étapes de l'histoire de la formation à distance dans les grands moments de l'histoire des sociétés occidentales (diffusion du christianisme, invention de l'imprimerie, révolution industrielle, émergence des médias de masse) pour ouvrir la discussion autour de dix grands " principes » qui, de son point de vue, ont régi et régissent ces modalités de formation. II engage les institutions qui projettent de poursuivre l'usage de la formation à distance au-delà de la pandémie actuelle à tenir compte de ces principes pour construire une offre de qualité.

C'est à propos d'une action particulière que l'article de Francis Bastien, sur « Les racines de FADIO : généalogie de la formation à distance dans l'Est-du-Québec », explique le rôle du contexte géographique 
et démographique, et le souci de répondre à des besoins socioéducatifs régionaux dans le processus qui a conduit à la mise en place de ce réseau de "Formation à distance interordres ",, identifié en 2020 par I'UNESCO comme « pratique prometteuse en éducation ».

L'histoire des institutions, quant à elle, est retracée à partir d'entretiens avec un des responsables (ou anciens responsables) de quatre universités à distance et d'une unité interne à une université traditionnelle. Ce sont, pour l'Europe, l'Open University britannique (OU), l'Université Ouverte de Catalogne (UOC) et l'unité Technologies éducatives et apprentissages (TECFA) de l'Université de Genève. Au Canada, les entretiens portent sur l'Université TÉLUQ au Québec, seule université à distance francophone au Canada, à l'histoire mouvementée (Guillemet, 2007) et sur l'Athabasca University, anglophone et, quant à elle, située en Alberta. Certaines de ces institutions sont relativement récentes, comme l'UOC, créée en 1990, tandis que d'autres existent depuis près d'une cinquantaine d'années. Leur histoire est donc longue, remplie d'événements tantôt bénéfiques, tantôt fâcheux. Ces entretiens sont précédés d'une introduction qui en souligne les points communs et les différences en termes d'origine, d'approches technopédagogiques et de perspectives d'avenir.

II ne reste qu'à vous souhaiter une bonne lecture de ces textes qui, nous n'en doutons pas, devraient susciter votre intérêt!

\section{Liste de références}

Acheré, C. (2014), Histoire de la Fédération Interuniversitaire de l'Enseignement à Distance (FIED). Distances et médiations des savoirs, (6). https://journals.openedition.org/dms/719

Albero, B. (2004). Technologies et formation : travaux, interrogations, pistes de réflexion dans un champ de recherche éclaté. Savoirs, 2(5), 9-69. https://www.cairn.info/revue-savoirs-2004-2-page-9.htm

Audet, L. (2012). Vingt-cinq ans d'apprentissage à distance au Canada francophone : profil, bilan et perspectives, Réseau d'enseignement francophone à distance du Canada. http://bv.cdeacf.ca/EA PDF/158154.pdf

Baron, G.-L. (2010). Quelles évolutions des professionnalités dans le contexte de l'enseignement supérieur en ligne? Quelques réflexions. Distances et savoirs, 8(2), 193-205. https://www.cairn.info/revue-distances-et-savoirs-2010-2-page-193.htm

Baron, G.-L. et Depover, C., (2019). Les effets du numérique sur l'éducation : Regards sur une saga contemporaine. Septentrion.

Benoist, P. (2004). Michel Debré et la formation professionnelle 1959-1971. Histoire de l'éducation, (101), 35-66. https://journals.openedition.org/histoire-education/725

Boissoneault, J. (2009). Enjeux de la médiatisation à l'université. Représentations dans la pratique professorale. Prise de Parole.

Bourrel, J.-R., Vidal, M. et Mahieux, F. (2008). L'histoire du CNED depuis 1939. Futuroscope Chasseneuil : CNED Direction générale.

Campus France. (2020). Le renouveau de l'enseignement à distance : panorama international et stratégies des établissements. Note $n^{\circ} 62$. https://ressources.campusfrance.org/publications/notes/fr/note $62 \mathrm{fr}$. pdf

Chaptal, A. (1995, juin). Technologies éducatives : des invariants à méditer. $1^{\text {res }}$ Rencontres de l'ORME, Marseille, France, p.15-18. https://edutice.archives-ouvertes.fr/edutice-00000345/document

Charlier, B., Deschryver, N. et Peraya, D. (2006). Apprendre en présence et à distance. Une définition des dispositifs hybrides. Distances et savoirs, 4(4), 469-496. https://www.cairn.info/revue-distances-et-savoirs-2006-4-page-469.htm

CLIFAD. (2007). Soixante ans de formation à distance au Québec. https://www.sofad.qc.ca/media/60_ans_fd.pdf

Cuban, L. (1986). Teachers and Machines: the Classroom Use of Technology since 1920. Columbia University Press.

Daniel, J. S. (1996). Mega-universities and Knowledge Media: Technology Strategies for Higher Education. Psychology Press. 
Depover, C. et Quintin, J.-J. (2011). Le tutorat et sa mise en œuvre. Dans C. Depover, B. De Lièvre, D. Peraya, J.-J. Quintin et A. Jaillet (dir.). Le tutorat en formation à distance (p. 39-54). De Boeck.

Depover, C., De Lièvre, B., Peraya D., Quintin, J.-J. et Jaillet, A. (dir.). (2011) Le tutorat en formation à distance. De Boeck.

Deschênes, A.-J. (1999). Un modèle de l'apprenant à distance : logique ou chaos. DistanceS, 3(2), 119-142, http://distances.teluq.ca/wp-content/uploads/2019/01/Distances-Vol-3-No2.pdf

Dessus, P. et Marquet, P. (2009). À la recherche des ingrédients actifs de l'apprentissage. Entretien avec Richard E. Clark. Distances et savoirs, 7(1), 113-124.

Dieuzeide, H. (1985). Les enjeux politiques. Dans F. Henri et A. Kaye (dir.), Le savoir à domicile : pédagogie et problématique de la formation à distance (p. 29-59). Presses de l'Université du Québec, Télé-université.

Glikman, V. (2016). La formation à distance en France, d'hier à aujourd'hui. Éducation permanente, (208), 137-156.

Glikman, V. (2002). Des cours par correspondance au « e-learning » : Panorama des formations ouvertes et à distance. Presses universitaires de France.

Glikman, V. (1999). Formations ouvertes et à distance : Le point de vue des usagers. Institut national de recherche pédagogique.

Glikman, V. et Baron, G.-L. (1991). Médias, multi-médias, technologies et formation à distance. Perspectives documentaires en éducation, (24), 63-94.

Guillemet, P. (2004). L'industrialisation de la formation, la fin d'un paradigme ? Distance et savoirs, 2(1), 93-118. https://www.cairn.info/revue-distances-et-savoirs-2004-1-page-93.htm

Guillemet, P. (2007). Former à distance. La Télé-université et l'accès à l'enseignement supérieur 1972-2006. Presses de l'Université du Québec.

Henri, F. (1985). La formation à distance : définition et paradigme. Dans F. Henri, F. et A. Kaye (dir.), Le savoir à domicile. Pédagogie et problématique de la formation à distance (p. 5-28). Québec, Canada, Presses Universitaires du Québec/Télé-université.

Jacquinot-Delaunay, G. et Fichez, É. (dir.) (2008). L'université et les TIC. Chronique d'une innovation annoncée. De Boeck. Lameul, G. et Loisy, C. (dir.) (2014). La pédagogie universitaire à l'heure du numérique. De Boeck.

Le Bars, S. (2020, 19 mai). Aux Etats-Unis, les étudiants ne veulent pas payer le prix fort pour des cours en ligne. Le Monde. https://tinyurl.com/f5eph2fv

Le Goff, J. (2014). Faut-il vraiment découper l'histoire en tranches? Le Seuil.

Mazouz, H. (2015). Citations et méditation : Au cœur de la réflexion. Edilivre.

Mœglin, P. (2016). Introduction. La question de l'industrialisation de l'éducation. Dans P. Mœglin (dir.), Industrialiser l'éducation : Anthologie commentée (1913-2012) (p. 9-73). Presses universitaires de Vincennes.

Papi, C. (2016). De l'évolution du métier d'enseignant à distance. STICEF, 23 ( $\mathrm{n}^{\circ}$ spécial), 15-45. http://sticef.univ-lemans.fr/num/vol2016/03-papi-ensaccapp/sticef 2016 NS papi 03p.pdf

Papi, C. (dir.) (2013). Le tutorat de pairs dans l'enseignement supérieur. Enjeux institutionnels, technopédagogiques, psychosociaux et communicationnels. L'Harmattan.

Poteaux, N. (2013). Pédagogie de l'enseignement supérieur en France : état de la question. Distances et médiations des savoirs, (4). https://journals.openedition.org/dms/403

Siemens, G. (2005). Connectivism: A Learning Theory for the Digital Age. International Journal of Instructional technology and distance learning, 2(1), 3-10. https://jotamac.typepad.com/jotamacs_weblog/files/Connectivism.pdf 